\title{
Enhancement of Dengue-2 E Protein Expression by the Expression of the Precursor Membrane Protein (Prm) of the Dengue-3 Virus
}

Michelle D de Oliveira ${ }^{1}$, André S de Oliveira ${ }^{1}$, Nina R Dutra ${ }^{1}$, Rafael F O França ${ }^{2}$, Eduardo R Honda ${ }^{3}$, Fernando B Zanchi ${ }^{3}$, Clovis A Neves ${ }^{1}$, Cynthia C da Silva1, Benedito A L da Fonseca ${ }^{4}$ and Sérgio O De Paula ${ }^{*}$

1Department of General Biology, Federal University of Viçosa, Minas Gerais, Brazil

2Department of Pharmacology, School of Medicine of Ribeirão Preto - University of Sao Paulo, Ribeirão Preto, Brazil

3Center of Medicine in Tropical Medicine (CEPEM), Rondônia, Brazil

4Department of Internal Medicine, School of Medicine of Ribeirão Preto - University of Sao Paulo, Ribeirão Preto, Brazil

\begin{abstract}
Several attempts to develop dengue recombinant subunit vaccines have failed due to insufficient levels of expression and incorrect folding of the $E$ protein. In order to verify the importance of the precursor membrane protein to the level of $\mathrm{E}$ protein expression, we constructed two recombinant plasmids by cloning the full-length sequence of the prM gene from the dengue-2 and dengue- 3 virus strains into a plasmid that expresses a truncated version of the $E$ protein of the dengue-2 virus. Next, we evaluated these two constructs for their effect on the levels of $E$ protein expression in vitro. Our results showed that both plasmids provided a correct expression of the $E$ protein as $E$ protein expression was detected in transfected Vero cells as demonstrated by indirect immunofluorescence and immunoblotting. Densitometry analysis of western blots of the cell extracts showed a $67.02 \%$ higher expression of $E$ protein in the cells transfected with pCID2EtD3prM, indicating that the prM sequence of the dengue-3 virus may be more effective in assisting with the correct processing of the $E$ protein. The results of this study may be used to increase the in vitro production of the $E$ protein antigen for use in vaccines and for diagnostic purposes.
\end{abstract}

Keywords: E protein; prM protein; Dengue; Vaccines; Chaperone

\section{Introduction}

Dengue fever is a major arthropod-borne viral disease caused by the dengue virus that affects humans. Approximately 2.5 million people live in areas that are considered to be at-risk for dengue fever, and every year occur 50 million infections. Nearly 500,000 dengue virus infections progress each year to more severe forms of the disease, such as hemorrhagic dengue fever [1]. However, there are no available vaccines or effective antiviral against the virus, and the treatment is mainly symptomatic.

There are four antigenically related serotypes [dengue-1, -2, -3, -4] that are known to cause dengue fever [2]. The dengue virions are spherical and icosahedral in shape with a positive sense RNA genome that has a single open reading frame (ORF) that codes for three structural proteins ( $\mathrm{C}$, prM and $\mathrm{E}$ ) that form the components of the virion and seven non-structural proteins (NS1, NS2A, NS2B, NS3, NS4A, NS4B and NS5) that are involved in the replication cycle of the virus $[3,4]$. The viral envelope has two transmembrane proteins, membrane protein $\mathrm{M}(8 \mathrm{kDa})$ and envelope glycoprotein $\mathrm{E}$ (51-60 $\mathrm{kDa}$ ), which forms homodimers that extend over the surface of the viral particle [5]. The interaction between the prM and $\mathrm{E}$ proteins is the main factor that directs the assembly of the virus [6]. In addition, during the replication cycle, the prM protein acts as a chaperone assisting in the correct folding of the E protein. After cleavage of the prM protein, the resulting peptide pr interacts with the E homodimers stabilizing them and preventing their disassembly [7].

During the virion assembly process, there is an interaction between the structural proteins prM and $\mathrm{E}$ that results in the formation of heterodimers in the endoplasmic reticulum (ER). This occurs in conjunction with the encapsidation of genomic RNA by capsid proteins and the aggregation of the prM and E proteins in the lipid bilayer of the ER to form immature virions. The prM protein is then cleaved to $M$ by furins or furin-type proteases in the trans-Golgi network and forming mature viral particles [6-9]. The $M$ protein remains in the mature viral particle as a transmembrane protein in the coating of $\mathrm{E}$ protein. This, in turn, undergoes structural changes that are triggered by low $\mathrm{pH}(\sim 5.8$ to 6.0$)$ and remains associated with the pr peptide, which prevents premature membrane fusion under low $\mathrm{pH}$ conditions $[3,7]$. This mechanism ensures that the fusion of viral and endosomal membranes occurs through an E protein-dependent mechanism under acidic conditions.

Both prM and E proteins are type I transmembrane proteins that contain a stem region and an anchor region within their C-termini. In the dengue virus, the stem and anchor regions of the prM and $\mathrm{E}$ proteins include two alpha-helices [8]. Previous studies have attempted to identify the key determinants for the interaction between these two proteins with the intention of defining a common assembly mechanism for flaviviruses; however, the interaction between the prM and $\mathrm{E}$ proteins is not completely understood $[6,8,10,11]$.

The E protein is responsible for cell receptor recognition and for the induction of neutralizing antibodies from the host. The E protein contains three domains that are composed mainly of $\beta$ sheets, referred to as domain I, which is the central domain, domain II, which is involved in dimerization and contains the fusion peptide, and domain III, which is involved in receptor binding [7,12]. Therefore, E protein has been an attractive candidate for its use in the development of an effective and safe recombinant vaccine $[13,14]$.

*Corresponding author: Sérgio Oliveira de Paula - Laboratory of Molecular Immu novirology, Federal University of Viçosa, Av. PH Rolfs, s/n., Viçosa, 36571-000, Minas Gerais, Brazil, Tel: +55-31-3899-2589/3899-2559; Fax: +55-31-3899-2549; E-mail: depaula@ufv.br

Received March 10, 2013; Accepted April 23, 2013; Published April 25, 2013 Citation: de Oliveira MD, de Oliveira AS, Dutra NR, França RFO, Honda ER, et al. (2013) Enhancement of Dengue-2 E Protein Expression by the Expression of the Precursor Membrane Protein (Prm) of the Dengue-3 Virus. J Vaccines Vaccin 4 182. doi: $10.4172 / 2157-7560.1000182$

Copyright: ( 2013 de Oliveira MD, et al. This is an open-access article distributed under the terms of the Creative Commons Attribution License, which permits unrestricted use, distribution, and reproduction in any medium, provided the original author and source are credited. 
In an attempt to develop an effective vaccine against dengue fever, the gene of the truncated $\mathrm{E}$ protein of the dengue virus-2 strain was inserted into the pCI vector without concomitant expression of prM. This method effectively reduced the size of the insert, then enabling the production of a quadrivalent vaccine in a single construct. The correct expression of the truncated $\mathrm{E}$ protein has been validated; however, it is expressed at a low level. Furthermore, there appears to be a robust and specific immune induction by the recombinant clone, but only a small percentage of mice that are immunized with the construct develop resistance to a subsequent challenge with the dengue-2 virus [15]. Based on these results, in this study we sought to improve the protective effect of this plasmid by inserting the prM genes of the dengue- 2 and dengue- 3 virus strains to determine the effect of prM expression on the expression of the E protein. After verifying the correct cloning and expression of the prM and E proteins, we observed an increase of $67.02 \%$ in the expression of the E protein when prM from the dengue-3 strain was expressed when compared with the prM gene from the dengue- 2 strain. In addition, we performed structural modeling and analysis of the prM proteins from both dengue strains, and we observed no significant differences between the two proteins. Our results suggest that the prM sequence of the dengue- 3 virus is a more effective chaperone resulting in an increase in the level of $\mathrm{E}$ protein expression. Our results offer a novel strategy to improve the expression of dengue subunit vaccines and should offer new insight into the development of dengue virus vaccines.

\section{Materials and Methods}

\section{Cell lines, viruses and plasmids}

C6/36 mosquito cells were grown at $28^{\circ} \mathrm{C}$ in Leibovitz medium (HIMEDIA) supplemented with $10 \%$ fetal calf serum. Vero cells were grown at $37^{\circ} \mathrm{C}$ under $5 \% \mathrm{CO}_{2}$ in MEM medium (Cultilab) supplemented with $10 \%$ fetal calf serum. The genes of interest were cloned using the plasmid pGEM-T easy (Promega Corporation, Madison, WI) and inserted into the $\mathrm{pCI}$ plasmid expression vector (Promega Corporation, Madison, WI) in which the gene for the truncated dengue-2 virus $\mathrm{E}$ protein had already been inserted. This plasmid was used in previous studies under the name pCID2Et [15]. The dengue-2 (New Guinea C strain) and dengue-3 (H87 strain) viruses were propagated in C6/36 cells in Leibovitz medium containing $2 \%$ inactivated fetal calf serum.

\section{Cloning of the prM genes}

The RNA of dengue- 2 and dengue- 3 viruses was purified from 500 $\mu \mathrm{L}$ of supernatant from $\mathrm{C} 6 / 36$ cell cultures infected with the respective virus using Trizol reagent (Invitrogen, Gaithersburg, MD) according to the manufacturer's instructions. RNA was reversed transcribed using the Superscript Kit (Invitrogen, USA). The prM protein genes were amplified in a PCR reaction using the following primers:

5'- CCCGAATTCTAGTGTCATTGAAGGAGCGACAG -3' and 5'- GGGCTCGAGATGGCATGATCATT-3' for prM/DENV2 and 5'-CCCGAATTCATCTGTCATGGATGGGGTAAC-3' and 5'-GGGCTCGAGTACACATCGCTCTGTCTCATG -3' for $\mathrm{prM} /$ DENV-3. PCR reactions were performed for 35 cycles under the following cycling conditions: $95^{\circ} \mathrm{C}$ for 1 minute, $55^{\circ} \mathrm{C}$ for 2 minutes and $72^{\circ} \mathrm{C}$ for 1 minute. The $540 \mathrm{bp}$ amplicons were separated on a $1.5 \%$ agarose gel and purified using the Wizard ${ }^{\oplus}$ SV Gel and PCR Clean-Up System (Promega Corporation, Madison, WI). Purified amplicons were ligated into the pGEM-T easy vector (Promega) using the enzyme T4 ligase (New England Biolabs, Beverly, MA) as recommended by the manufacturer. Plasmids that had the correct inserts were sequenced using the MegaBACE ${ }^{\mathrm{TM}} 500$ sequencer (GE Healthcare, England). The concentrations of plasmids used for sequencing were of 75 to $100 \mathrm{ng} /$ $\mu \mathrm{L}$ and the concentrations of the specific primers were $2.5 \mathrm{ng}$ in a final volume of $3 \mu \mathrm{L}$.

\section{Cloning of the prM genes in the pCID2Et expression plasmid}

The pGEM-T plasmids containing the prM genes were digested with XhoI and EcoRI restriction enzymes at the same time as the pCID2Et plasmid. The prM/DENV-2 and prM/DENV-3 genes were then cloned separately into expression vector pCID2Et using T4 ligase, according to the manufacturer's instructions (New England Biolabs, Beverly, MA). These constructs were named pCID2EtD2prM and pCID2EtD3prM (Figure 1).

\section{Analysis of the expression of the prM and $\mathrm{E}$ proteins in vitro}

Vero cells were transiently transfected with recombinant plasmids using calcium phosphate precipitation and chloroquine [16]. Briefly, the medium on cultures of confluent Vero cells was refreshed two hours before the transfection procedure and maintained at $37^{\circ} \mathrm{C}$ in the presence of $5 \% \mathrm{CO}_{2}$. Two different concentrations of the recombinant plasmids pCID2EtD2prM and pCID2EtD3prM and the control plasmid pCI were used ( $30 \mu \mathrm{g}$ and $50 \mu \mathrm{g})$. For each concentration, 60 $\mu \mathrm{L}$ of $2 \mathrm{M} \mathrm{CaCl}_{2}$ and TE buffer (10 mM Tris- $\mathrm{HCl}, 10 \mathrm{mM}$ EDTA 1; $\mathrm{pH}$ 8.0) was added to achieve a final volume of $480 \mu \mathrm{L}$. In a second tube for each reaction, $480 \mu \mathrm{L}$ of $2 \mathrm{X}$ HEBS buffer $(\mathrm{NaCl} 280 \mathrm{mM}$; HEPES $50 \mathrm{mM} ; \mathrm{Na}_{2} \mathrm{HPO}_{4} 1.5 \mathrm{mM}$; $\mathrm{pH}$ 7.2) was added. The contents of both tubes were mixed slowly under gentle agitation and incubated for 45 minutes. The mixture was added to Vero cell along with $200 \mu \mathrm{L}$ of chloroquine. The cells were incubated for 5 hours at $37^{\circ} \mathrm{C}$ and after one wash with $1 \mathrm{X}$ PBS, fresh culture medium was added. The cells were incubated for either $48 \mathrm{~h}$ or $72 \mathrm{~h}$ at $37^{\circ} \mathrm{C}$ with $5 \% \mathrm{CO}_{2}$. Then, the cells were analyzed for the expression of the $\mathrm{E}$ and prM proteins through immunofluorescence assays, immunoprecipitation and real-time PCR to detect the levels of mRNA.

\section{SDS-PAGE and immunoblotting}

Cell extracts from the transfected cells were immunoprecipitated using polyclonal anti-dengue serum as the primary antibody followed by separation by $10 \%$ SDS-PAGE [16]. After separation, the proteins were transferred to a nitrocellulose membrane, which was blocked overnight with PBS/Tween $0.5 \% / 3 \%$ casein. Then, the membrane was washed twice with PBS/Tween $0.5 \%$ (PBS-T) and incubated for 2 hours at room temperature with agitation in the presence of human antidengue antibody diluted at 1:100 in PBS (immunized human serum). After two more washes with PBS-T, the membrane was incubated for two hours at room temperature with gentle agitation in the presence of anti-human $\operatorname{IgG}$ antibody conjugated with horseradish peroxidase (Sigma-Aldrich, Germany). Membranes were developed using the

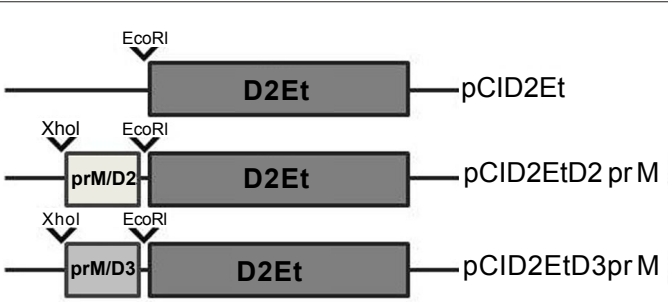

Figure 1: Schematic showing the constructs used in this study, pCID2EtD2prM and pCID2EtD3prM, after cloning the prM/DENV-2 and prM/DENV-3 genes into the expression vector pCID2Et. 
FAST $^{\mathrm{TM}}$ DAB peroxidase substrate (Sigma-Aldrich, Germany), as recommended by the manufacturer. Protein bands were subjected to densitometry analysis using the Image J software (Image Analyzing Software, Germany).

\section{Indirect immunofluorescence (IFA)}

Vero cells were transfected as previously described. Seventy-two hours post-transfection, cells were washed twice with 1X PBS for 5 minutes and fixed in cold acetone for 15 minutes. The cells were incubated with mouse ascitic fluid clonal antibodies (MIAF) against the E protein of dengue-2 diluted at 1:10 and kept at $37^{\circ} \mathrm{C}$ for 1 hour. After three washes with $1 \mathrm{X}$ PBS for 5 minutes each, the antigen-antibody complexes were detected with $30 \mu \mathrm{L}$ of anti-mouse IgG antibody labeled with FITC at a dilution of 1:200 in 1X PBS. After a 1 hour incubation for at $37^{\circ} \mathrm{C}$, the cells were washed three times with $1 \mathrm{X}$ PBS for 5 minutes each and analyzed using a fluorescence microscope. With the aid of the Image-Pro Plus 4.0 software (Media Cybernetics, USA) the fluorescence intensity of FITC in images was analyzed. The cells analyzed were delineated with the magic wand tool (range: 20; smooth: 1 ), and FITC intensity was plotted in the histograms. The X-axis indicates the intensity of green (FITC emission range) and ranges from 0 to 255, whereas the Y-axis indicates the number of pixels with certain intensity and ranged from 0000 to 1000 .

\section{Real-time polymerase chain reaction}

RNA was extracted from cells transfected with recombinant plasmids and with the pCI control plasmid as previously described. Briefly, $2 \mu \mathrm{g}$ of cDNA from the cell lysates was used in a $25-\mu \mathrm{L}$ reaction that included $2 \mu \mathrm{M}$ of each primer for the respective prM proteins and $12.5 \mu \mathrm{L}$ of Mastermix SyBr Green PCR (Fermentas). The reaction conditions were as follows: $95^{\circ} \mathrm{C}$ for 10 minutes followed by 40 cycles of $95^{\circ} \mathrm{C}$ for 1 minute, $55^{\circ} \mathrm{C}$ for 2 minutes and $72^{\circ} \mathrm{C}$ for 2 minutes. Quantification of the expression of each transcript was performed using the software Gene Amp ${ }^{\circledR} 5700$ Sequence Detection System Version 1.3 (Applied Biosystems). As an endogenous control, the $\beta$-actin gene was used to normalize the transcript levels.

\section{Molecular modeling}

To investigate possible structural differences that might be associated with different levels of $\mathrm{E}$ protein expression, we performed prM/DENV-3 homology modeling using the crystal structure of the precursor membrane protein (prM) of the dengue- 2 virus envelope as a template (code PDB: 3C5X C chain) with a $2.2 \AA$ resolution [17]. To find the prM/DENV-3 sequence, we used the NCBI website (Table 1). To find and obtain the template structure, we used the database of protein structure Brookhaven (PDB). The alignment of sequences was performed using the MODELLER v9.9 [18] and ClustalW software. The modeling by homology was performed using MODELLER v9.9 software. A total of 1000 templates were generated and the final template was selected based on having the lowest energy conformation as calculated by the DOPE application in the MODELLER software. The overall stereochemical quality of the end of the prM/DENV-3 template was evaluated using the PROCHECK software [19]. The interactive view and comparative analysis of structures were performed using the Swiss-PDB viewer 4.0.2 software [20]. The images of the structures were viewed using the POV-ray 3.62 software.

\section{Results}

\section{Sequencing}

To confirm the correct cloning, the prM genes of the pCID2EtD2prM and pCID2EtD3prM plasmids were sequenced and the sequences were analyzed using BLASTn and BLASTx software. We also performed a comparative analysis between the two nucleotide sequences through the "BLAST two sequences" software to determine the degree of identity between the two sequences and a comparative analysis between the two amino acid sequences of the prM genes using the "ClustalW" software (Figures 2A-2C). For the prM protein of the dengue- 2 virus strain (prM/DENV-2) insert, there was 98\% identity between the nucleotide sequence and the corresponding sequence in the wild type DENV-2, and $1 \%$ of the sequence was gapped. The amino acid sequence of our clone showed $97 \%$ identity with the precursor membrane prM/ DENV-2 protein and no gaps were present (Figure 2A). Sequencing
A

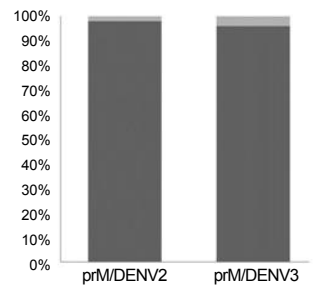

B

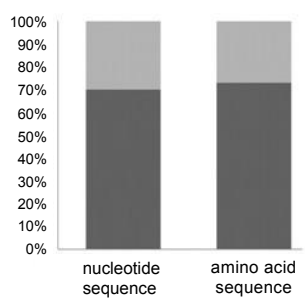

C

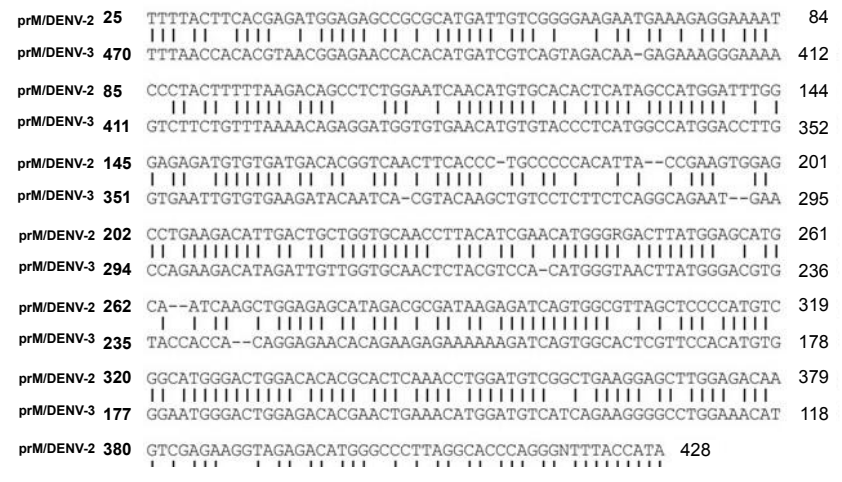

Figure 2: (A) Sequences of the cloned inserts in both recombinant clones demonstrating the identity compared to the published genome for the dengue virus. (B) Comparative analysis between the two nucleotide sequences and between the two amino acid sequences for detecting the degree of identity. (C) Alignment of the sequences (prM/DENV-2 and prM/DENV-3) to detect the degree of identity between the two sequences. This alignment was performed using the "BLAST two sequences" software.

\begin{tabular}{|l|l|l|}
\hline Insert & BLASTn & BLASTx \\
\hline prM/DENV-2 & $\begin{array}{l}\text { gb/AF038403.1/AF038403. Identities 473/479 (98\%) Gaps } \\
5 / 479(1 \%) .\end{array}$ & $\begin{array}{l}\text { refNP739582.2 (Membrane glycoprotein precursor Dengue virus 2). } \\
\text { GENE ID: 1494449 DENV gp1 (Polyprotein Dengue virus 2). Identities 97/100 (97\%) Positives } \\
98 / 100(98 \%) \text { Gaps 0/100. }\end{array}$ \\
\hline prM/DENV-3 & $\begin{array}{l}\text { emb/AJ563355.1/DVI563355. Identities 541/533 (96\%) } \\
\text { Gaps 12/533 (2\%) }\end{array}$ & $\begin{array}{l}\text { gb/ACL99113.1 (Polyprotein Dengue virus 3), gb/ACL99114.1 (Polyprotein Dengue virus 3). } \\
\text { Identities 76/130 (58\%) Positives 86/130 (66\%) Gaps (1/130) 0\% }\end{array}$ \\
\hline
\end{tabular}

Table 1: BLASTn nucleotide alignment and BLASTx translated sequence alignment of the prM/DENV-2 and prM/DENV-3 inserts. 
and alignment of the prM protein of the dengue-3 virus strain prM/ DENV-3 insert revealed $96 \%$ identity with the corresponding wild type sequence of DENV-3, and $2 \%$ of the sequence was gapped. The amino acid sequence of our clone showed $58 \%$ identity with the precursor polyprotein of wild type DENV-3 with no gaps (Table 1). Aligning both of our clones against one another revealed $70 \%$ identity between the $\mathrm{prM} / \mathrm{DENV}-2$ and prM/DENV-3 proteins with $3 \%$ gaps and an E-value of $2^{\mathrm{e}-43}$. Alignment of the amino acid sequences of our cloned inserts showed $73 \%$ identity with no gaps and an E-value of $9^{\mathrm{e}-28}$ (Figure $2 \mathrm{~B}$ ).

\section{Expression of the truncated E protein and pCID2EtD2prM} and pCID2EtD3prM plasmids

To evaluate the expression of the truncated E protein by our plasmids, Vero cells were transfected with $30 \mu \mathrm{g}$ and $50 \mu \mathrm{g}$ pCID2EtD2prM or pCID2EtD3prM plasmid or an empty pCI plasmid. The cell extracts were separated using SDS-PAGE and visualized by silver staining. As shown in figure $3 \mathrm{~A}$, a band was detected with a molecular weight of

A

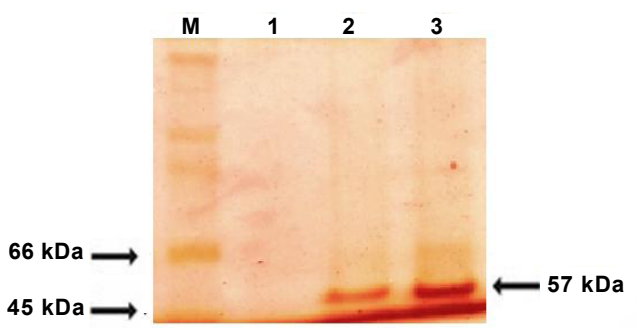

B

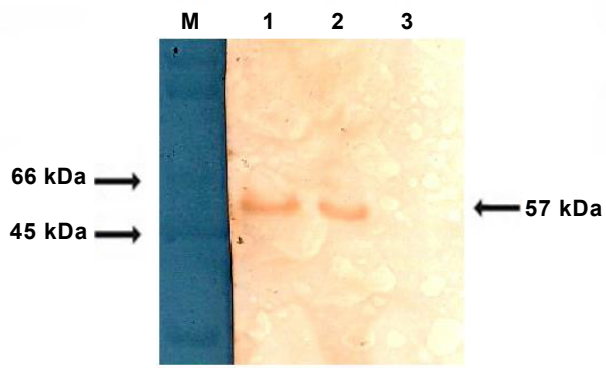

C

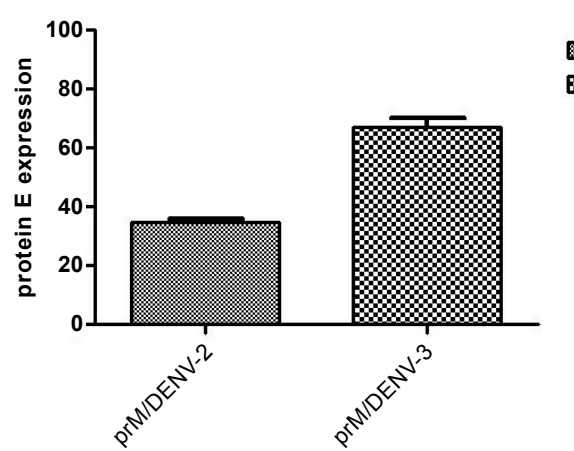

Figure 3: (A) Electrophoresis in a $10 \%$ polyacrylamide gel showing (from left to right) the molecular weight marker ("Molecular Weight Marker", Sigma-Aldrich, Germany), negative control of an immunoprecipitate of cells transfected with the pCID2EtD2prM construct and an immunoprecipitate of cells transfected with the pCID2EtD3prM construct. (B) Imunoblot showing (from left to right) the molecular weight marker ("Molecular Weight Marker", Sigma-Aldrich, Germany), an immunoprecipitate of cells transfected with the pCID2EtD2prM construct and an immunoprecipitate of cells transfected with the pCID2EtD3prM construct and a negative control of an immunoprecipitate of a cell extract from cells transfected with $\mathrm{pCl}$ vector. (C) Result of optical densitometry analysis of $\mathrm{E}$ protein expression (western blot densitometry).

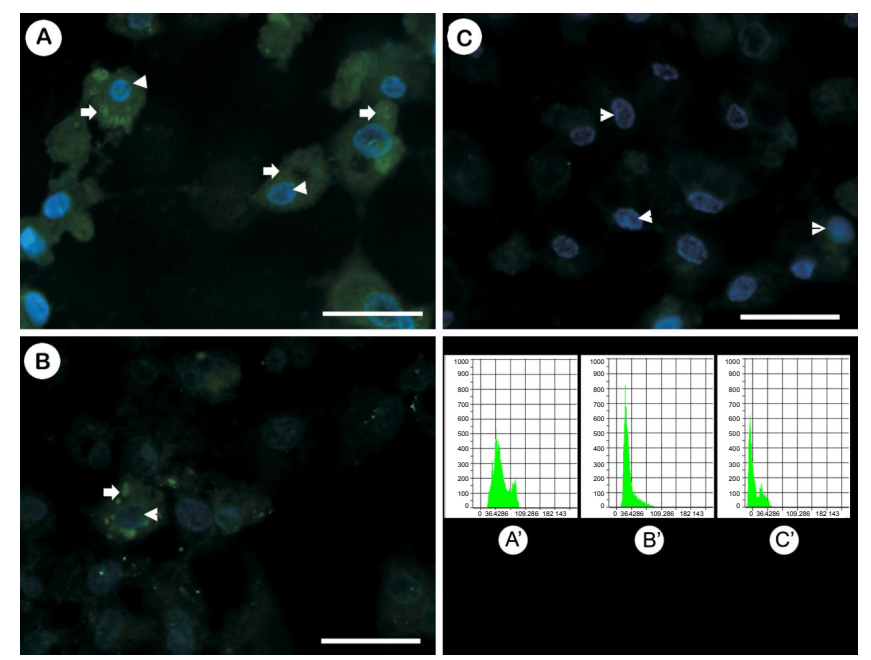

Figure 4: Detection of viral E proteins by IFA (arrows) in transfected Vero cells and DAPI for nuclear contrast (arrow heads). (A) Positive immunofluorescence for expression of the dengue virus $E$ protein (transfected with pCID2EtD3prM), (B) Positive immunofluorescence for dengue virus $\mathrm{E}$ protein expression (transfected with pCID2EtD2prM), (C) Negative immunofluorescence for dengue virus (transfected with $\mathrm{pCl}$ ). Bar $=30 \mu \mathrm{m}$. Histograms quantitating the fluorescence intensity for dengue virus $E$ protein in Vero cells. Figure $A$ ': transfection with pCID2EtD3prM, B ': transfection with pCID2EtD2prM. C ': negative immunofluorescence (transfection with $\mathrm{pCl}$ ). The X-axis indicates the fluorescence intensity, whereas the $Y$-axis indicates the number of pixels that demonstrate a given intensity.

approximately $57 \mathrm{kDa}$, which corresponds to the expected molecular weight of the truncated E protein. To confirm the E protein expression by our constructs, an immunoblot was performed using cell extracts of transfected Vero cells. The results of the immunoblot revealed a $57 \mathrm{KDa}$ band supporting the idea that the truncated E protein of dengue virus-2 is efficiently expressed from the pCID2EtD2prM and pCID2EtD3prM plasmids (Figure 3B). We observed a higher level of expression for the pCID2EtD3prM clone. The protein bands obtained were analyzed using optical densitometry obtained with ImageJ software (Image Analyzing Software, Germany). Densitometry analysis revealed that the protein product of prM/DENV-3 increased the expression of the truncated protein $\mathrm{E}$ by $67.02 \%$ when compared with the protein product of prM/DENV-2 (Figure 3C). Cells that were transfected with $50 \mu \mathrm{g}$ of either the pCID2EtD3prM or the pCID2EtD2prM clone resulted in a positive immunofluorescence IFA demonstrating that the $\mathrm{prM} / \mathrm{E}$ proteins are expressed (Figures 4A-4C). Cells that were transfected with pCID2EtD3prM (Figure A') showed more fluorescent signal than cells that were transfected with either the pCID2EtD2prM plasmid (Figure B') or a control plasmid (Figure C').

\section{Expression of prM proteins by recombinant plasmids}

To analyze the efficiency of prM gene transcription in transfected cells, we performed a real time PCR reaction using the transcriptome of cell extracts. The results showed an increase in the amount of messenger RNA after 12 hours of transfection with a greater increase in cells that were transfected with the plasmid pCID2EtD3prM (data not shown). These results are consistent with the previous results and strongly suggest that the prM/DENV-3 insert provides a much higher level of expression of the truncated $\mathrm{E}$ protein by the dengue- 2 virus when compared with the prM/DENV-2 insert (data not shown).

\section{Molecular modeling and structural analysis}

Homology modeling is usually the method of choice when there is 
a clear homology ratio between the sequence of the target protein and at least one known structure. This approach produces reasonable results based on the assumption that the tertiary structures of two proteins are similar as long as their sequences are related [21].

To assess the validity of $3 \mathrm{C} 5 \mathrm{X}$ as a structural model for prM/DENV3 , we examined the sequence homology. Alignment revealed $74 \%$ identity between prM/DENV-3 and 3C5X (Figure 5). Figure 6 shows the Ramachandran plot [22] for the final prM/DENV-3 model. The percentage of residues resting in the favored regions of a Ramachandran plot is one of the best parameters to verify the stereochemical quality of a protein model, assuming that a good model should have more than $90 \%$ of residues in allowed regions [19].

The quality of the structural model for prM/DENV-3 was also evaluated by comparing the predicted structure with the 3C5X model structure through the superposition of the atoms and the evaluation of the mean square deviation. Figure 7 shows the superposition of our proposed prM/DENV-3 structure and the prM/DENV-2 template (PDB code: 3C5X C chain). The root mean square (RMS) deviation of $\mathrm{C}$ a between the homologous structure (prM/DENV-3) and the model is $0.21 \AA$, which encourages the validity of our proposed structure and suggests similarity between our structure and the model structure $3 \mathrm{C} 5 \mathrm{X}$.

\section{Discussion}

E protein plays important roles during the life cycle of the dengue virus, including cell receptor binding and entry into the host cell via membrane fusion. An unusual feature of this protein is that its activities are regulated through the interaction with another viral protein, the prM protein [11]. The $\mathrm{prM} / \mathrm{pr}$ protein acts as a chaperone assisting the correct conformational folding of the E protein and binds to the tip of the $\mathrm{E}$ protein. In addition, prM undergoes a conformational change under low $\mathrm{pH}$ conditions to allow the rearrangement of $\mathrm{E}$ protein on the surface of the virus and allows access to furin processing. After furin cleavage, the pr peptide remains associated with the $\mathrm{E}$ protein preventing it from inducing premature fusion and stabilizing the $\mathrm{E}$ protein homodimers and preventing the dissociation of $\mathrm{E}$ protein monomers [3,7].

Studies on dengue virus and other flavivirus have shown that the coexpression of the prM protein is necessary for the correct $\mathrm{E}$ protein expression $[15,23]$. Some studies have suggested that the concomitant expression of the prM and $\mathrm{E}$ proteins of the dengue- 3 virus results in higher levels of $\mathrm{E}$ protein expression than coexpressing the prM and $\mathrm{E}$ proteins in dengue-2 virus [24-26]. These results suggest that protein prM of the dengue- 3 serotype is more effective in assisting processing of the E protein. In addition, Guirakoo and colleagues have demonstrated that for the Murray Valley encephalitis virus, virions containing non-processed prM protein were more acid-resistant than those that contained the processed mature form of prM. This suggests that prM

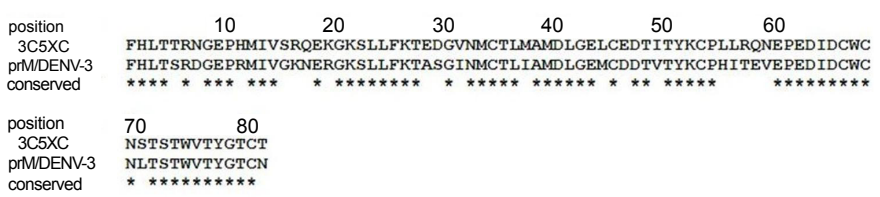

Figure 5: Alignment performed using the MODELLER software. $3 C 5 \mathrm{X}$ is the sequence of the mold structure of the prM protein of the dengue- 2 virus ( $C$ Chain - code PDB: 3C5X). prM/DENV-3 served as the target sequence. The alignment resulted in $74 \%$ similarity as calculated using ClustalW software.

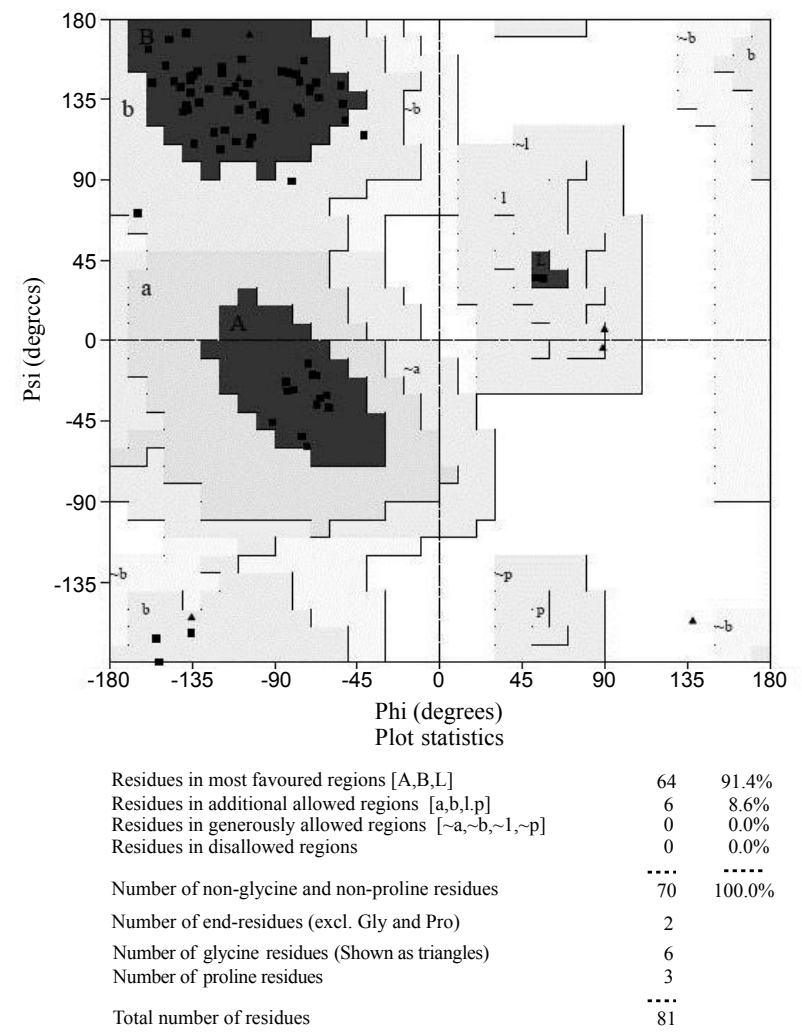

Figure 6: Ramachandran plot for the modeled structure of prM/DENV-3. The analysis shows that $91.4 \%$ of the amino acids are in energetically favorable conformations.

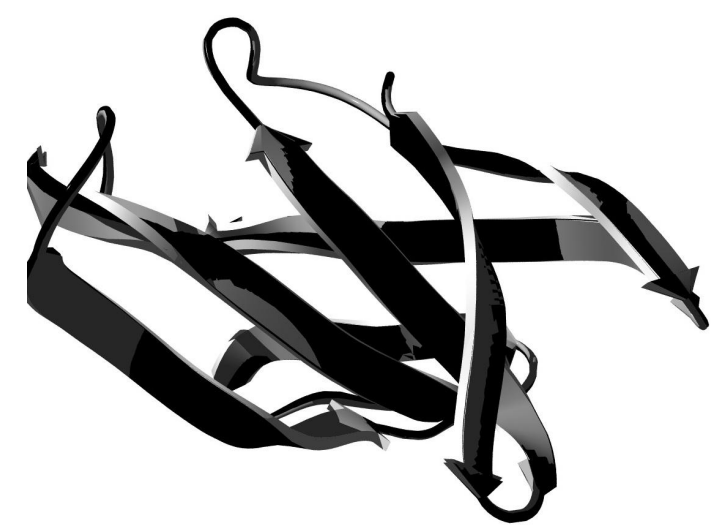

Figure 7: Structural superposition of the model and target structures. Ribbon representation as executed using the Swiss-PDB Viewer and rendered with the POV-ray. The mold structure of the prM protein of the dengue- 2 virus is presented in black (C Chain - code PDB: 3C5X) and the structure model is presented in gray (prM/DENV-3). The RMSD between both carbons is $0.21 \AA$.

protein prevents irreversible conformational changes in protein $\mathrm{E}$ during the maturation of the virions under acidic conditions [26].

Ocazionez and Fonseca have proposed that a possible reason for the low level of protection conferred by DNA vaccine against DENV-2 was a weak immune system activation resulting from the insufficient secretion of the truncated E protein due to a lack of prM protein [15]. To investigate this hypothesis, the genes of the prM protein of dengue-2 and dengue- 3 viruses were inserted into the pCID2Et plasmid, which expresses the truncated E protein of the dengue-2 virus. The 
plasmids created were called pCID2EtD2prM and pCID2EtD3prM, respectively.

The sequences of the inserts displayed a high degree of identity with the respective wild type genes. For pCID2EtD2prM, we observed $98 \%$ identity with the wild type dengue- 2 prM sequence, and for pCID2EtD3prM, we observed 96\% identity with the wild type dengue-3 prM sequence. The prM/DENV-2 insert had $97 \%$ identity with the wild type amino acid sequence and $98 \%$ similarity, while for the prM/DENV3 insert, the amino acid sequence identity and similarity were $76 \%$ and $86 \%$, respectively, between residues when compared to the wild type sequence. An alignment between the sequences of the dengue- 2 and dengue- 3 prM proteins revealed that $71 \%$ of their nucleotide sequences were identical. Furthermore, alignment of the amino acid sequences from both serotypes revealed $70 \%$ identity and $84 \%$ similarity. An alignment of the sequences of the inserts of the prM proteins of the dengue- 2 and dengue- 3 serotypes revealed $70 \%$ identity in the nucleotide sequences. Alignment of the translated sequences revealed $75 \%$ identity and $91 \%$ similarity between the protein sequences. These data show that prM/DENV-2 and prM/DENV-3 proteins expressed by the recombinant plasmids have greater sequence similarity among them than that found between the prM proteins of DENV-2 and DENV-3.

Both constructs were capable of expressing the $\mathrm{E}$ protein in Vero cells. Vero cells were transfected with either of the constructs (pCID2EtD2prM and pCID2EtD3prM) and cell extracts were collected. Cell extracts were separated by SDS-PAGE analysis, which revealed a protein band of $56-57 \mathrm{KDa}$, which is the expected size of the $\mathrm{E}$ protein. These results were confirmed by an immunoblot, in which bands corresponding to the $\mathrm{E}$ protein were observed. A more evident band was observed for samples that were transfected with the pCID2EtD3prM construct. Densitometry analysis revealed a $67.02 \%$ increase in the E protein expression level in samples transfected with the plasmid containing the prM/DENV-3 insert. This suggests that the prM protein of the dengue- 3 virus is a more efficient chaperone for $\mathrm{E}$ protein processing than the prM protein of dengue-2. The expression of the structural E protein of the DENV-2 was also verified through indirect immunofluorescence. Vero cells transfected with the pCI vector displayed no fluorescence, whereas cells that were transfected with 50 $\mu \mathrm{g}$ of either recombinant plasmid displayed significant fluorescence.

Based on these results, it is possible to infer that the prM protein of the dengue- 3 virus is more efficient in assisting with $\mathrm{E}$ protein processing. The increased rate of $\mathrm{E}$ protein expression may be the result of increased cleavage of prM/DENV-3 compared with prM/DENV-2, which would result in a greater degree of protection from E-proteindependent premature membrane fusion. This would culminate in an increase in the secretion of the E protein.

The interactions between prM and E guide the assembly and secretion of the viral particle. During the translation of the viral polyprotein, prM is the first protein to be translocated into the lumen of the endoplasmic reticulum and subsequently acts as a chaperone for the proper folding of the E protein. Moreover, cleavage by furin, which is present in the trans-Golgi, releases the $\mathrm{N}$-terminal pr peptide, which remains attached to the terminus of domain II of the E protein and protects it from premature fusion under the acidic conditions found in the cellular secretory pathway $[7,17,27]$. Thus, the prM/DENV-3 protein may have cleavage sites that are more accessible to furin, which would facilitate formation of the pr protein resulting in protection of the $\mathrm{E}$ protein from immature fusion and resulting in higher expression levels of E protein. Differences in the cleavage pattern of prM have been identified between the prM proteins of different flaviviruses
[27]. In this study, the authors tested the influence of the amino acid residues located near the furin cleavage site. The resultant engineered viruses showed that the prM protein of the Japanese encephalitis virus was more susceptible to cleavage, whereas the prM protein from the tick-borne encephalitis virus was more resistant to cleavage by furin. However, for the strains of dengue virus analyzed in this study, there was no difference in the consensus sequence required for processing by furin in the Golgi (Arg-Xaa-(Lys/Arg)-Arg) or in the proximal residues that may favor furin cleavage. The constructs in the present study may show the importance of the proximal residues because it has been previously reported that the amount of basic residues present favors the interaction with furin [27].

Differences between the residues crucial for interaction between the prM protein and the E protein may also be important. The residues $\mathrm{Asp}^{63}$ and $\mathrm{Asp}^{65}$ of the prM protein have been shown to interact with the $\mathrm{His}^{244}$ residue of the E protein $[7,17]$. The dengue-2 prM insert codes for an asparagine residue at this position, while the same position in the dengue-3 prM insert codes for a threonine residue. This indicates that, although prM/DENV-2 may have a greater affinity for the E protein of the same serotype, the prM/DENV-3 protein may be more readily processed and thus more effective at protecting the $\mathrm{E}$ protein from premature fusion during the exocytic cellular pathway. This would lead to a higher level of protein $\mathrm{E}$ detection. However, more experiments are necessary to determine which residues are crucial to explain the differences revealed by our study.

Recently, several studies have attempted to increase the expression of the E protein of the dengue virus in DNA vaccines or in heterologous systems with the goal of producing antigens on a large scale for diagnostic purposes and/or vaccines. Our findings provide a novel method to increase the expression level of the E protein, which can be used to help develop vaccines and other treatments for dengue fever.

\section{Acknowledgements}

This work was supported by Conselho Nacional de Desenvolvimento Científico e Tecnológico (CNPq), Brazil (Pronex Grant \# 550165/2010-0 and INCT Dengue Grant \# 471221/2008-2), FAPERO and FAPEMIG. MDO was supported by a scholarship from the CAPES/Brazilian Nanobiotech Network Project n. 8.

\section{References}

1. Guzman MG, Halstead SB, Artsob H, Buchy P, Farrar J, et al. (2010) Dengue: a continuing global threat. Nat Rev Microbiol 8: S7-16.

2. Halstead SB. Dengue. Lancet 370: 1644-1652.

3. Perera R, Kuhn RJ (2008) Structural proteomics of dengue virus. Curr Opin Microbiol 11: 369-377.

4. Costa SM, Azevedo AS, Paes MV, Sarges FS, Freire MS, et al. (2007) DNA vaccines against dengue virus based on the ns1 gene: the influence of different signal sequences on the protein expression and its correlation to the immune response elicited in mice. Virology 358: 413-423.

5. Zybert IA, van der Ende-Metselaar H, Wilschut J, Smit JM (2008) Functiona importance of dengue virus maturation: infectious properties of immature virions. J Gen Virol 89: 3047-3051.

6. Tan TT, Bhuvanakantham R, Li J, Howe J, Ng ML (2009) Tyrosine 78 of premembrane protein is essential for assembly of West Nile virus. J Gen Virol 90: 1081-1092.

7. Zheng A, Umashankar M, Kielian M (2010) In vitro and in vivo studies identify important features of dengue virus pr-E protein interactions. PLoS Pathog 6 : e1001157

8. Lin YJ, Peng JG, Wu SC (2010) Characterization of the GXXXG motif in the first transmembrane segment of Japanese encephalitis virus precursor membrane [prM] protein. J Biomed Sci 17: 39.

9. Junjhon J, Edwards TJ, Utaipat U, Bowman VD, Holdaway, et al. (2010) 
Citation: de Oliveira MD, de Oliveira AS, Dutra NR, França RFO, Honda ER, et al. (2013) Enhancement of Dengue-2 E Protein Expression by the Expression of the Precursor Membrane Protein (Prm) of the Dengue-3 Virus. J Vaccines Vaccin 4: 182. doi:10.4172/2157-7560.1000182

Influence of pr-M cleavage on the heterogeneity of extracellular dengue virus particles. J Virol 84: 8353-8358

10. Lin YJ, Wu SC (2005) Histidine at residue 99 and the transmembrane region of the precursor membrane prM protein are important for the prM-E heterodimeric complex formation of Japanese encephalitis virus. J Virol 79: 8535-8544

11. Wang S, He R, Anderson R (1999) PrM- and cell-binding domains of the dengue virus E protein. J Virol 73: 2547-2551.

12. Whitehead SS, Blaney JE, Durbin AP, Murphy BR (2007) Prospects for a dengue virus vaccine. Nat Rev Microbiol 5: 518-528.

13. De Paula SO, Lima DM, de Oliveira Franca RF, Gomes-Ruiz AC, Fonseca BA (2008) A DNA vaccine candidate expressing dengue-3 virus prM and $E$ proteins elicits neutralizing antibodies and protects mice against lethal challenge. Arch Virol 153: 2215-2223

14. Perez-Velez ME, Garcia-Nieves T, Colon-Sanchez C, Martinez I (2009) Induction of neutralization antibodies in mice by Dengue-2 envelope DNA vaccines. P R Health Sci J 28: 239-250.

15. Ocazionez Jimenez R, Lopes da Fonseca BA (2000) Recombinant plasmid expressing a truncated dengue-2 virus $\mathrm{E}$ protein without co-expression of prM protein induces partial protection in mice. Vaccine 19: 648-654.

16. Sambrook JF, E F Maniatis T (1989) Molecular Cloning. A Laboratory Manual, Cold Spring Harbor New York.

17. Li L, Lok SM, Yu IM, Zhang Y, Kuhn RJ, et al. (2008) The flavivirus precursor membrane-envelope protein complex: structure and maturation. Science 319 : 1830-1834.

18. Sali A, Blundell TL (1993) Comparative protein modelling by satisfaction of spatial restraints. J Mol Biol 234: 779-815.
19. Laskowski RA, MacArthur MW, Moss DS, Thornton JM (1993) PROCHECK: program to check the stereochemical quality of protein structures. Journal of Applied Crystallography 26: 283-291.

20. Guex N, Peitsch MC (2005) SWISS-MODEL and the Swiss-PdbViewer: an environment for comparative protein modeling. Electrophoresis 18: 2714-2723.

21. Kroemer RT, Doughty SW, Robinson AJ, Richards WG (1997) Prediction of the three-dimensional structure of human interleukin-7 by homology modeling Protein Eng 9: 493-498.

22. Ramachandran GN, Ramakrishnan C, Sasisekharan V (1963) Stereochemistry of polypeptide chain configurations. J Mol Biol 7: 95-99.

23. Fonseca BA, Pincus S, Shope RE, Paoletti E, Mason PW (1994) Recombinan vaccinia viruses co-expressing dengue-1 glycoproteins prM and $E$ induce neutralizing antibodies in mice. Vaccine 12: 279-285.

24. Blair PJ, Kochel TJ, Raviprakash K, Guevara C, Salazar M, et al. (2006) Evaluation of immunity and protective efficacy of a dengue-3 pre-membrane and envelope DNA vaccine in Aotus nancymae monkeys. Vaccine 24: 14271432.

25. Konishi E, Yamaoka M, Kurane I, Mason PW (2000) A DNA vaccine expressing dengue type 2 virus premembrane and envelope genes induces neutralizing antibody and memory B cells in mice. Vaccine 18: 1133-1139.

26. Guirakhoo F, Kitchener S, Morrison D, Forrat R, McCarthy K, et al. (2006) Live attenuated chimeric yellow fever dengue type 2 [ChimeriVax-DEN2] vaccine: Phase I clinical trial for safety and immunogenicity: effect of yellow fever preimmunity in induction of cross neutralizing antibody responses to all 4 dengue serotypes. Hum Vaccin 2: 60-67.

27. Keelapang P, Sriburi R, Supasa S, Panyadee N, Songjaeng A, et al. (2004) Alterations of pr-M cleavage and virus export in pr-M junction chimeric dengue viruses. J Virol 78: 2367-2381. 